\title{
Evaluation of the effect of BMSCs condition media and methylprednisolone in TGF- $\beta$ expression and functional recovery after an acute spinal cord injury
}

\author{
Khoshsirat $\mathrm{S}^{1}$, Abbaszadeh $\mathrm{HA}^{1,2,6}$, Ahrabi B ${ }^{2}$, Bahrami $\mathrm{M}^{2}$, Abdollahi far $\mathrm{MA}^{2}$, Khoramgah $\mathrm{MS}^{3}$, \\ Roozbahany NA ${ }^{1,4}$, Darabi $\mathrm{S}^{5}$
}

Hearing Disorders Research Center, Shahid Beheshti University of Medical Sciences, Tehran, Iran.

Dr.abbaszadeh79@gmail.com

\begin{abstract}
The purpose of this experimental study is evaluation of the effect of BMSCs Condition Media and Methylprednisolone in TGF- $\beta$ expression and functional recovery after acute spinal cord injury in adult wistar rat.

MATERIAL AND METHODS: After an acute spinal cord injury, MP and BMSC-CM were injected intraperitoneally and the recovery rate was evaluated by BBB and narrow beam test. Real time PCR, H\&E staining, cavity formation and stereology was done after 12 weeks post injury.

RESULT: There were significant differences in functional recovery between MP+CM group, relative to other groups. TGF- $\beta 1$ expression decreased in MP+CM group compared to the control group. Cavity size in experimental groups decreased compared to the control group. The mean volume of spinal cord demonstrated a significant increase in the $\mathrm{MP}+\mathrm{CM}$ group in comparison to the other experimental groups.

CONCLUSION: Simultaneous use of MP and BMSC-CM will improve recovery from the spinal cord injury, reduce inflammation and improve functional recovery (Tab. 1, Fig. 8, Ref. 26). Text in PDF www.elis.sk.

KEY WORDS: spinal cord injury, TGF- $\beta 1$, inflammation, BMSC, methylprednisolone.
\end{abstract}

\section{Introduction}

Spinal cord injuries (SCI) are usually caused by severe mechanical trauma to the spinal cord and may lead to severe motor dysfunctions, cardiovascular problems, venous thrombosis, osteoporosis, pressure ulcers, and neuropathic symptoms (1). SCI cause a break in the blood-brain barrier, release of inflammatory factors and activation of glial cells that ultimately cause a spinal cord necrosis (2). Initial disruption of blood vessels following SCI leads to the development of micro hemorrhage in Gray and white matter and happens after injury that causes necrotic change and ischemia, inflammation, apoptosis, distraction of blood-spinal cord barrier and finally neurological dysfunction and increasing tissue loss $(3,4,5)$. Along with a secondary cell death, the inflammatory

${ }^{1}$ Hearing Disorders Research Center, Loghman Hakim Hospital, Shahid Beheshti University of Medical Sciences, Tehran, Iran, ${ }^{2}$ Department of Biology and Anatomical Sciences, Shahid Beheshti University of Medical Sciences, Tehran, Iran, ${ }^{3}$ Department of Biotechnology, School of Advanced Technologies in Medicine, Shahid Beheshti University of Medical Sciences, Tehran, Iran, ${ }^{4} \mathrm{G}$. Raymond Chang School, Ryerson University, Toronto, Canada, ${ }^{5}$ Cellular and Molecular Research Center, Qazvin University of Medical Science, Qazvin, Iran, and ${ }^{6}$ Skull Base Research Center, Loghman Hakim Hospital, Shahid Beheshti University of Medical Sciences, Tehran, Iran

Address for correspondence: H.A. Abbaszadeh, Dr, Hearing Disorders Research Center, Shahid Beheshti University of Medical Sciences, Tehran, Iran. reaction takes place. After SCI, neutrophils and macrophage migrate to the site of the injury through damaged blood brain barrier $(6,7,8,9)$. Increasing of TGF- $\beta 1$ may exert its Pro-inflammatory reactions through direct and indirect after SCI.

TGF- $\beta$ has a key role in adaptable cell growth, differentiation and immune cells function (10). It can inhibit the proliferation of immune active cells and lymphocyte differentiation. It facilitates the synthesis of extracellular matrix and can modulate progression of fibrosis via decreasing the synthesis of extracellular matrix-degrading enzymes, such as matrix metalloproteinase (MMP) and also by enhancing expression of proteinase inhibitor. Prescription of medications that decrease inflammatory infiltration and can inhibit the injury expansion(11). Methylprednisolone (MP) is a corticosteroid that inhibit lipid peroxidation and reduces inflammatory cell and cytokine at the site of injury and improve the perfusion of the spinal cord and reduce the influx of neuronal calcium $(12,13,14)$ The purpose of this experimental study is evaluation of the effect of BMSCs Condition Media and MP in TGF- $\beta$ expression and functional recovery after acute spinal cord injury in adult wistar rat.

\section{Matrials and methods}

Animals

All the action plans in this study were examined and accepted by the Ethics Committee on Animal Experimentation of the Shahid 
Beheshti University of Medical Sciences (IR.SBMU.RETECH. REC.1396.1284, Tehran, Iran). A total of 35 female Wistar rats $(180-200 \mathrm{~g})$ were used in the current study (32 rats for rat model experimental groups and 3 for cell preparations). We kept the rats in our animal care facility (temperature $\left(20^{\circ} \mathrm{C}\right)$ and a $12 / 12$ hour light-dark cycle) of the Biology and Anatomical science Department of Shahid Beheshti University of Medical Sciences (15).

\section{Isolation and culture of Bone marrow stem cells (BMSCs)}

Bone marrow was aspirated from femur bones of three healthy adult Wistar rats by a syringe needle $(18 \mathrm{G})$ containing $4 \mathrm{ml}$ of Dulbecco's Modified Eagle Medium-low glucose (DMEM, Gibco, USA) and was supplemented with $10 \%$ fetal bovine serum (FBS, Gibco, USA), $1 \%$ penicillin/streptomycin, and then cultured in 25 $\mathrm{cm}^{2}$ flask at $37^{\circ} \mathrm{C}$ in a water-saturated atmosphere of $5 \% \mathrm{CO}_{2} / 95$ $\%$ air condition. Non-adherent cells were discarded at $24 \mathrm{~h}$ after initial harvesting. The culture media (DMEM/ FBS 10\%) was replaced twice a week, and cells were passaged after reaching $70 \%$ confluence $(15,16)$. BMSCs were analyzed by flowcytometry for mesenchymal markers (CD44, CD45, CD33, CD106).

\section{Osteogenic and adipogenic differentiation}

For differentiation of BMSCs into osteogenic and adipogenic cells, the cells were incubated in differentiation media for 21 days. Differentiation capacity of these cells was evaluated by Alizarin red and oil red staining.

\section{BMSC-CM preparation}

BMSCs in third passages were processed for BMSC-CM collection. Cultures were washed twice with phosphate-buffered saline (PBS) (Sigma-Aldrich, USA), when harvested BMSCs confluency reached $\sim 90 \%$. than $4 \mathrm{~mL}$ of DMEM supplemented with $0 \%$ FBS and $1 \%$ antibiotics, so called serum-free culture medium, was added to every flask. The conditioned medium was collected after incubation with BMSC for 48 hours. The resulted BMSC-CM undergone sterile filtration and was stored at $-80^{\circ} \mathrm{C}$ until use (15).

\section{qRT-PCR}

To determine the abundance of transcripts of genes including TGF- $\beta$, we collected the whole RNA from removal spine at 1 week using RNA Extraction kit (Invitrogen, USA) Based on the company's protocol. cDNAs were produced by the Prime Script TM RT reagent Kit (Fermentas, Lithuania). Reverse transcription was carried out Step OnePlus ${ }^{\mathrm{TM}}$ Real-Time PCR System (Applied Biosystems, USA) with Syber Green PCR Master Mix (Applied Biosystems, USA) based on the company's guidelines under the following conditions: initial denaturation at $95^{\circ} \mathrm{C}$, denaturation at $95^{\circ} \mathrm{C}$ for $30 \mathrm{sec}$, annealing at $56^{\circ} \mathrm{C}$ for $45 \mathrm{sec}$, extension at $72^{\circ} \mathrm{C}$ for $45 \mathrm{sec}$ and a final polymerization at $72^{\circ} \mathrm{C}$ for $10 \mathrm{~min}$. The primer sequences are brief in table 1 . We applied the comparative a $\mathrm{Ct}$ method for gene target expression analysis $(2 \Delta \Delta \mathrm{Ct})(17)$. All samples normalized with GAPDH level, as the loading control (16).

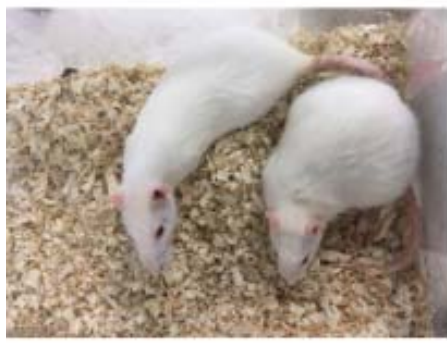

Adult SD RAT

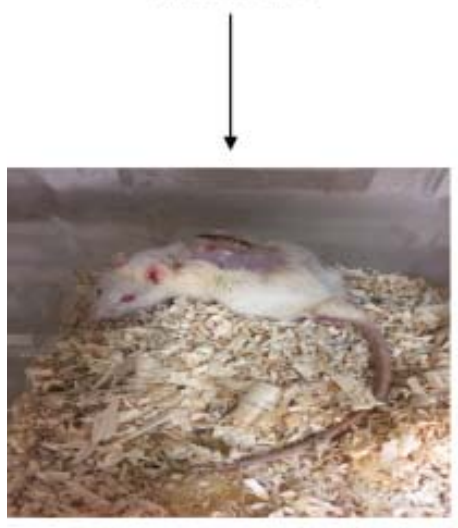

Contusion model

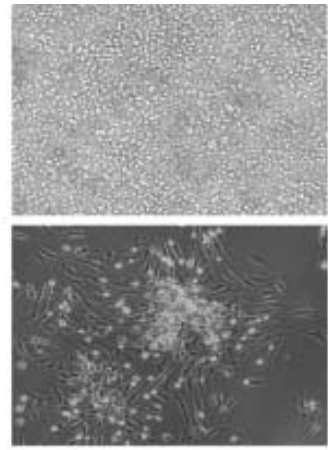

BMSCs aspiration

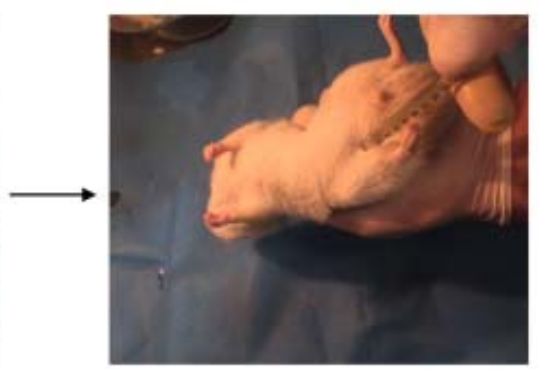

$\mathrm{CM}+\mathrm{MP}$ injection

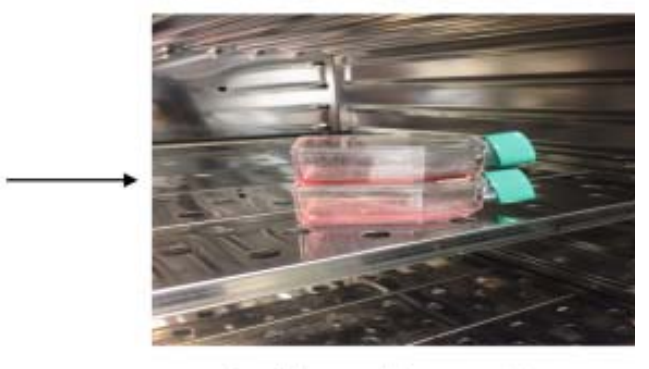

Condition media preparation

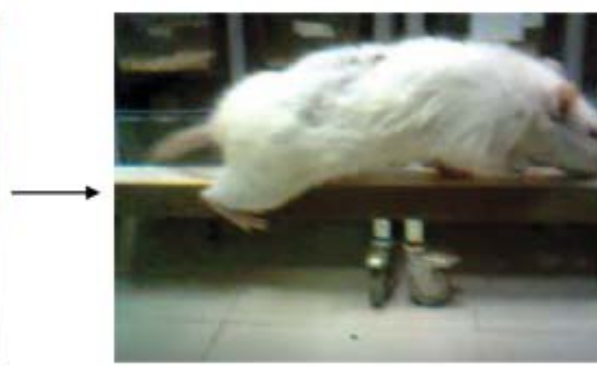

Functional recovery test 
$684-691$

Tab. 1. Primers Sequence used in the qRT-PCR.

\begin{tabular}{|c|c|c|c|c|}
\hline GENE & Primer type & Sequence & Annealing temperature & Product size (Base pairs) \\
\hline TGB- $\beta$ & $\begin{array}{l}\mathrm{F} \\
\mathrm{R}\end{array}$ & $\begin{array}{c}\text { AAGAAGTCACCCGCGTGCTA } \\
\text { TGTGTGATGTCTTTGGTTTTGTCA }\end{array}$ & 61 & $180 \mathrm{bp}$ \\
\hline GAPDH & $\begin{array}{l}\mathrm{F} \\
\mathrm{R}\end{array}$ & $\begin{array}{c}\text { CCACAACTC TTCCATTCTC } \\
\text { CCAAGATTCACGGTAGATAC }\end{array}$ & 59 & $200 \mathrm{bp}$ \\
\hline
\end{tabular}

\section{Surgical procedures}

Adult female rats were deeply euthanized by intraperitoneally (IP) injection of $80 \mathrm{mg} / \mathrm{kg}$ ketamine and $10 \mathrm{mg} / \mathrm{kg}$ diazepam (Sigma-Aldrich, USA) and were placed in prone position. Skin, subcutaneous tissue, and muscles were incised in the midline at the T9-T10 level and laminectomy was performed in these levels, without disrupting the dura. After the stabilization of the spine with clamping spinous processes of $\mathrm{T} 8$ and $\mathrm{T} 11$, the contusion model of SCI was performed by the use of a metal bar weighted
$10 \mathrm{~g}$ which was dropped from the height of $25 \mathrm{~mm}$ to the posterior surface of the region of spinal cord. Then the surgical site was washed by normal saline and the skin and muscles of the lesion area were sutured. The animals were held in the box for recovery. After surgery, rats received antibiotic for 7 days and sedative for 2 days. In order to facilitate urinary excretion and prevent bladder dysfunction, the bladder of rats emptied by massaging three time a day (15). We randomly divided the animal into four groups containing eight rats in each group: contusion only as control group,
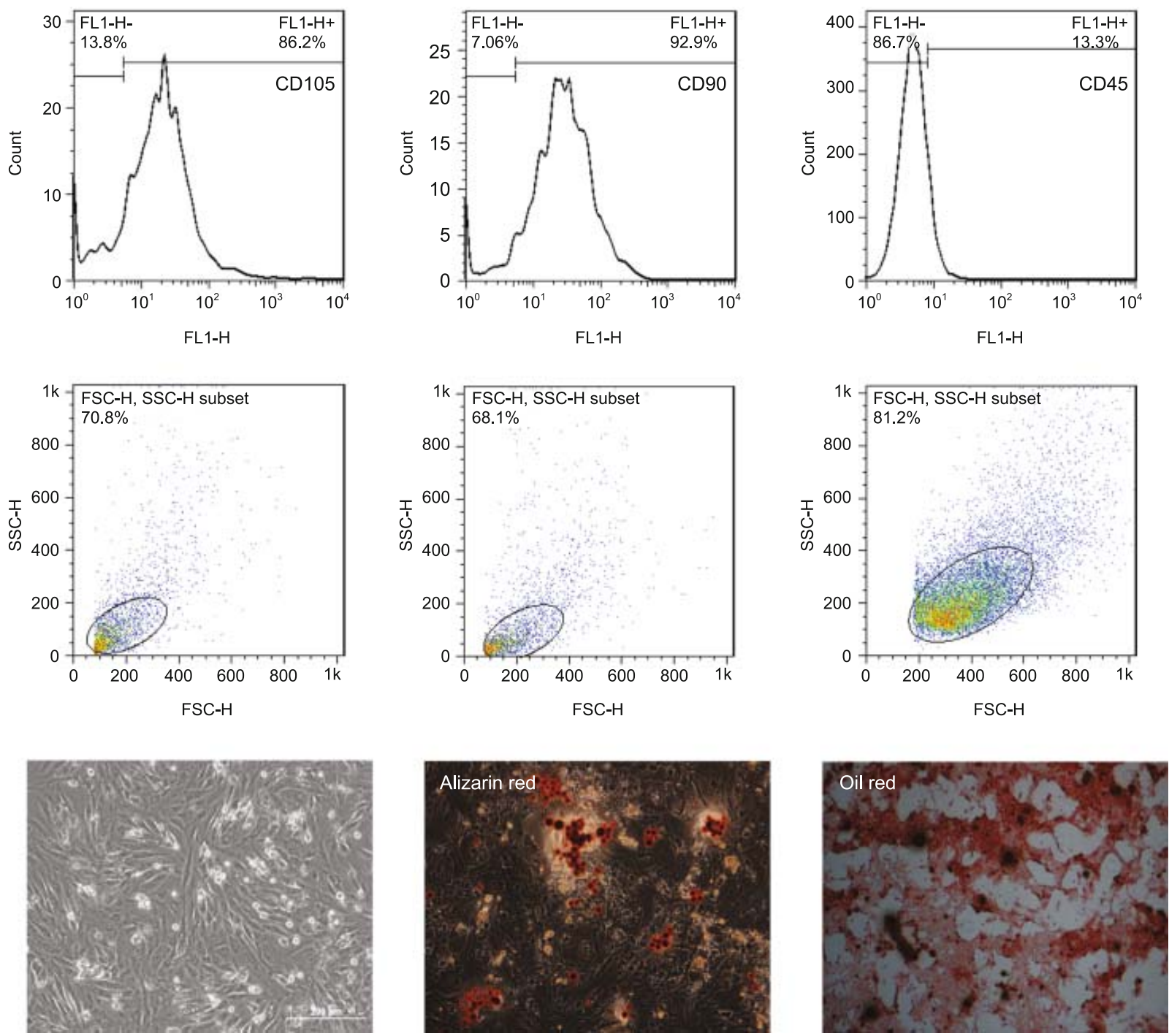

Fig. 1. Morphology, Adipogenic and osteogenic differentiation in BMSC (Lower row). Flowcytometry CD marker analysis of BMSC shows were positive for CD44, CD90, CD73 and negative for CD45, CD34 (Upper row). 
contusion+ MP (Pharmacia; $30 \mathrm{mg} / \mathrm{kg}$ i.v, Intramuscular injection), contusion $+\mathrm{CM}$ and contusion $+\mathrm{MP}+\mathrm{CM}$.

\section{$B B B$ open field locomotion scoring}

Behavioral tests were done weekly using the Basso-BeattieBresnahan (BBB) Locomotors Scale by two investigators, who were blinded to treatment groups' status. All behavioral tests began $1 \mathrm{~d}$ after the injury and then continued once per week for 12 weeks.

\section{Narrow beam scoring}

Narrow beam walking test was done to estimate sensory-motor functional recovery following SCI. Briefly; we trained the rats to cross a narrow wooden beam ( $80 \mathrm{~cm}$ long and $4 \mathrm{~cm}$ wide) for 5 days ( 5 min per day). Narrow beam test began 1 day after SCI (15).

\section{Hematoxylin and Eosin (H\&E) staining\& Stereological analysis \& Morphometric Assessment}

After sacrificing all the rats on week 13 after surgery, their spinal cords were procured and fixed in $4 \%$ paraformaldehyde for the period of $12 \mathrm{~h}$, then they were processed using the automatic tissue processor (Leica TP 1020; Leica, Hamburg, Germany) and embedded in paraffin (Sigma-Aldrich). Serial sections of 7- $\mu \mathrm{m}$ section thickness were made and then deparaffinized with xylol (Sigma-Aldrich). H\&E staining was performed on sections of deparaffinized tissue. The mean cavity size was obtained according to Cavalieri's principle. The total volume of the spared tissue was obtained by series summation of the spared tissue and cavity volumes. The cavity volume was obtained by using the formula: $\mathrm{Vsp}=\mathrm{a} \times \mathrm{d}$, where " $a$ " is the measured area, and " $\mathrm{d}$ " is the intersection distance.

\section{Statistical analysis}

Tukey's post hoc test and One Way ANOVA were used for statistical analysis. The data are presented as mean \pm SEM. P-values

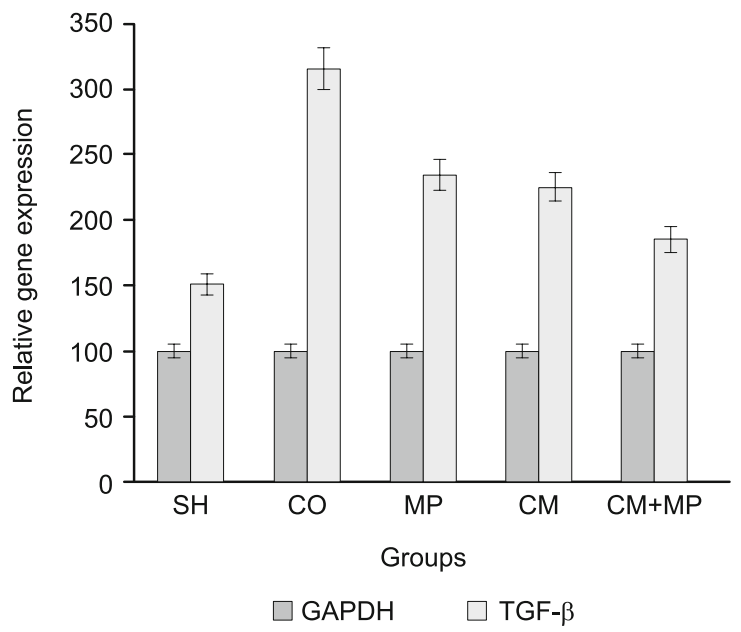

Fig. 2. The relative quantitative expression of TGF- $\beta$ using real time RT-PCR as compared to rat spinal cord.* $p<0.05$ (mean \pm SEM). Laminectomy: sham group, CO: contusion only as control group, MP: contusion + MP (Pharmacia; $30 \mathrm{mg} / \mathrm{kg}$ i.v. intramuscular injection), $\mathrm{CM}$ : contusion $+\mathrm{CM}$ and $\mathrm{MP}+\mathrm{CM}$ : contusion $+\mathrm{MP}+\mathrm{CM}$.
$<0.05$ is considered as statistically significant. We used IBM SPSS 22.0 software for our statistical analysis(16).

\section{Results}

\section{BMSCs characterization}

Osteogenic and adipogenic differentiation of BMSCs (passage4) was detected by alizarin red and oil red staining 21day after incubation by differentiation media (Figure1). The CD markers expression were also analyses using flowcytometry. The result of flowcytometry showed that the expression of BMSCs CD markers such as CD105, CD90 is positive (86.2\%, 92.9\% respectively) and CD45 low expressed (Figure1).

\section{Real time PCR analysis}

The results indicated that the spinal cord transcribed TGB- $\beta$ mRNAs expression decrease in MP, CM, MP/CM compared to the control group, respectively (Fig. 2).

\section{$B B B$ result}

BBB test were assessed for a $12 \mathrm{~W}$ period following the contusion model induction in the experimental groups. The mean BBB scores of control and experimental groups revealed that the scores were gradually increased from post injury induction. Functional recovery significantly in the $\mathrm{MP}+\mathrm{CM}$ and at low levels in the $\mathrm{CM}$ groups in the $12 \mathrm{~W}$ post injury in comparison with that of the control groups. The score of the MP+CM group was significantly higher than the scores of other experimental groups in W8-W12. $*(\mathrm{P}<0.05)$. (Fig. 3).

\section{Narrow beam test}

Before the injury, rats had no difficulty traveling across the beam and had no errors in placing theirs foots, but after the injury, they were passing across the narrow beam with difficulty and they gave a poor performance (Fig. 4). However, their performance crossing the beam ameliorated gradually six weeks after the surgery.

\section{$H \&$ E staining}

The results of H\&E staining after 12 weeks after lesion showed that the structure of white matter and gray matter in the laminectomy group did not change, while in the control group, the number of neurons lowered and a large cavity was observed. In other experimental groups (MP, CM, MP+CM), different degrees of tissue destruction and cavity formation were observed. Cell density was more in the cavity in the control group than in the experimental group.

Levels of microglial and inflammatory cells in the control group were higher than in the other groups around the cavity. Replacement of myelin and white matter structure in the experimental group improved. Cavity size in the experimental groups decreased compared to the control group (Fig. 5).

\section{Stereological analysis \& Morphometric Assessment}

The results of the screening of histological sections 12 weeks after the injury for the evaluation of cavity formation in differ- 


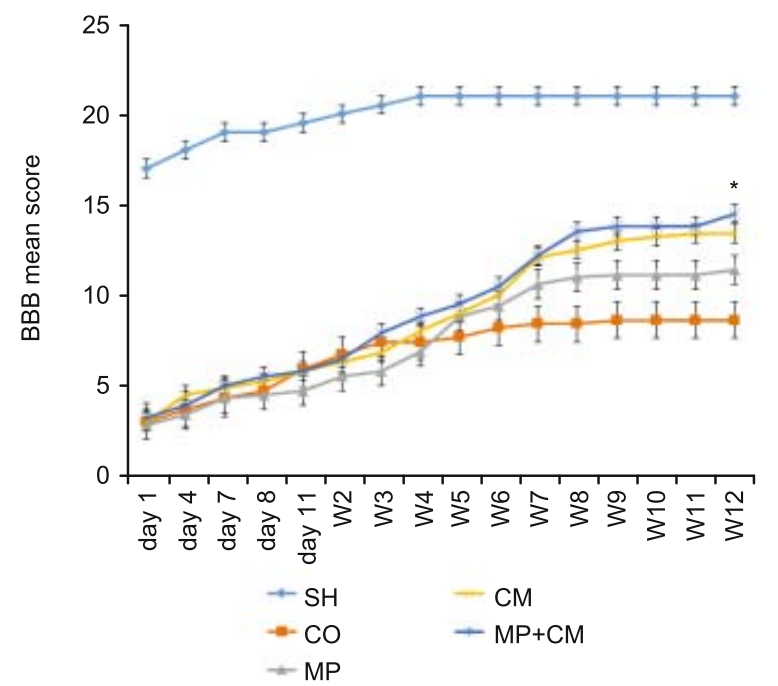

Fig. 3. BBB locomotor rating scores in all the experimental groups. Following the spinal cord injury induction, the scores were evaluated over a 12-week period. The scores of the MP+CM group were significantly higher than those of other experimental groups in W8-W12 (*p $<$ 0.05). SH: sham group, CO: contusion only as control group, MP: contusion + MP (Pharmacia; $30 \mathrm{mg} / \mathrm{kg}$ i.v. intramuscular injection), CM: contusion + CM and MP + CM: contusion + MP + CM.

ent groups with Image J software, version 1.46r, showed that the cavity was not formed in the laminectomy group and there was a significant difference in the other groups, but the formation of the cavity in the CM/MP group was lower than in the other groups .Cavity formation in the group $\mathrm{CM}$ had a significant difference with MP group (Fig. 6).

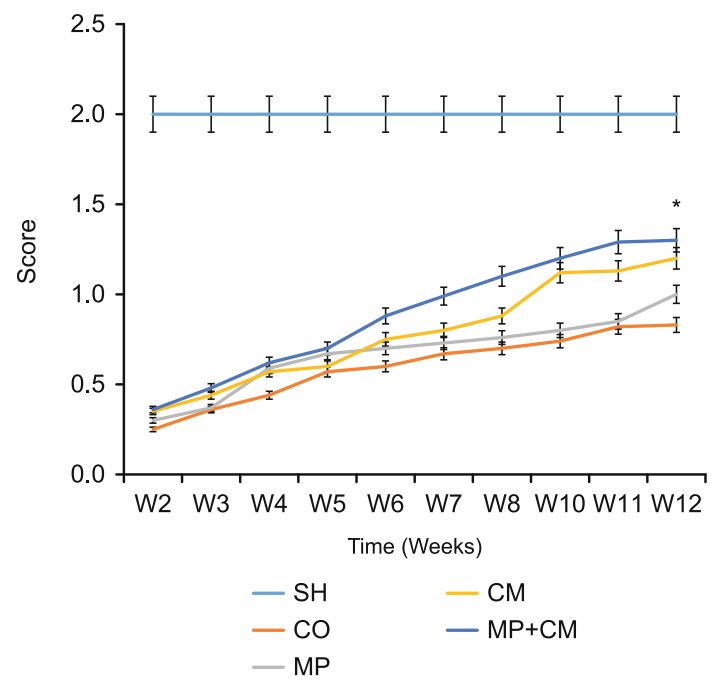

Fig. 4. Narrow beam test. Sensorimotor test represented a higher function recovery in the implanted group as compared to the control group at week 10 (*p< $<05)$. SH: sham group, CO: contusion only as control group, MP: contusion + MP (Pharmacia; $30 \mathrm{mg} / \mathrm{kg}$ i.v. intramuscular injection), CM: contusion $+\mathrm{CM}$ and MP $+\mathrm{CM}$ : contusion $+\mathrm{MP}+\mathrm{CM}$.

\section{Estimation volume of spinal cord}

The results showed that the mean volume of spinal cord had a significant increase in the $\mathrm{MP}+\mathrm{CM}$ group in comparison with the other groups (MP,CM,CO) $(* \mathrm{p}<0.05)$ (Fig. 7).

\section{Estimation number of neurons and glial cells in spinal cord}

The results showed that the total number of the glial cells was significantly higher in the control group than in the other experi-
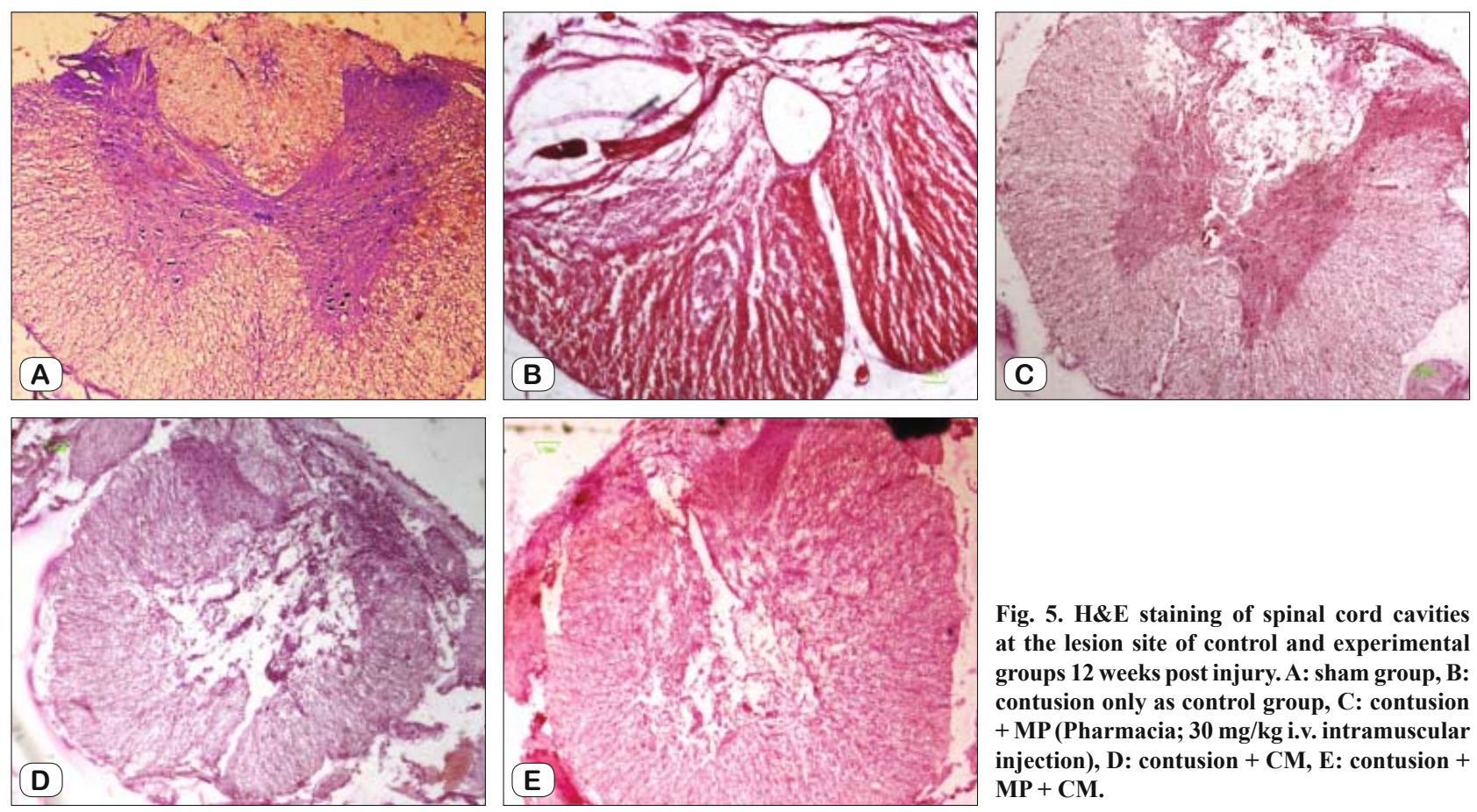

Fig. 5. H\&E staining of spinal cord cavities at the lesion site of control and experimental groups 12 weeks post injury. A: sham group, B: contusion only as control group, $C$ : contusion + MP(Pharmacia; $30 \mathrm{mg} / \mathrm{kg}$ i.v. intramuscular injection), D: contusion + CM, E: contusion + $\mathbf{M P}+\mathbf{C M}$. 


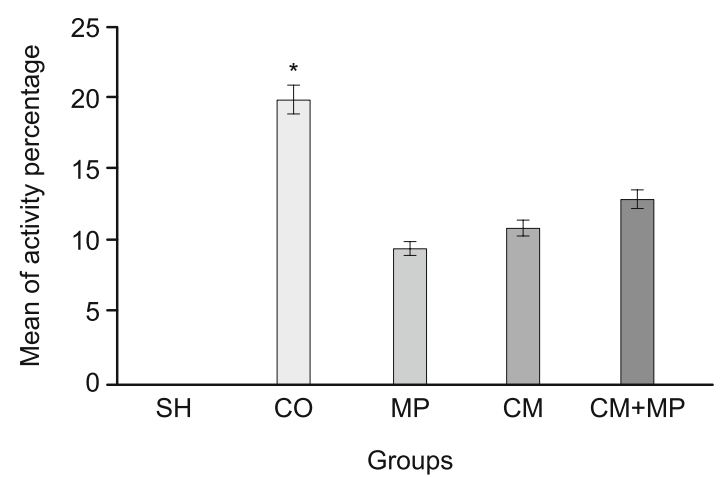

Fig. 6. Mean cavitation percentages in all the experimental groups. Contusion, MP, CM, MP + CM. The results showed that the cavity formation in the contusion group was higher than in the other groups and in the MP+CM group this amount decreased significantly (*p < 0.05). SH: sham group, CO: contusion only as control group, MP: contusion + MP (Pharmacia; $30 \mathrm{mg} / \mathrm{kg}$ i.v. intramuscular injection), CM: contusion + CM and MP + CM: contusion + MP + CM.

mental groups (MP, $\mathrm{CM}, \mathrm{MP}+\mathrm{CM})$, and the number of neurons in posterior and anterior horns in the $\mathrm{MP}+\mathrm{CM}$ group was higher than in the other groups (Contusion, CM, MP) $(* \mathrm{p}<0.05)$ (Fig. 8).

\section{Discussion}

Spinal cord injuries are usually caused by severe mechanical trauma to the spinal cord and cause cardiovascular problems, ve-
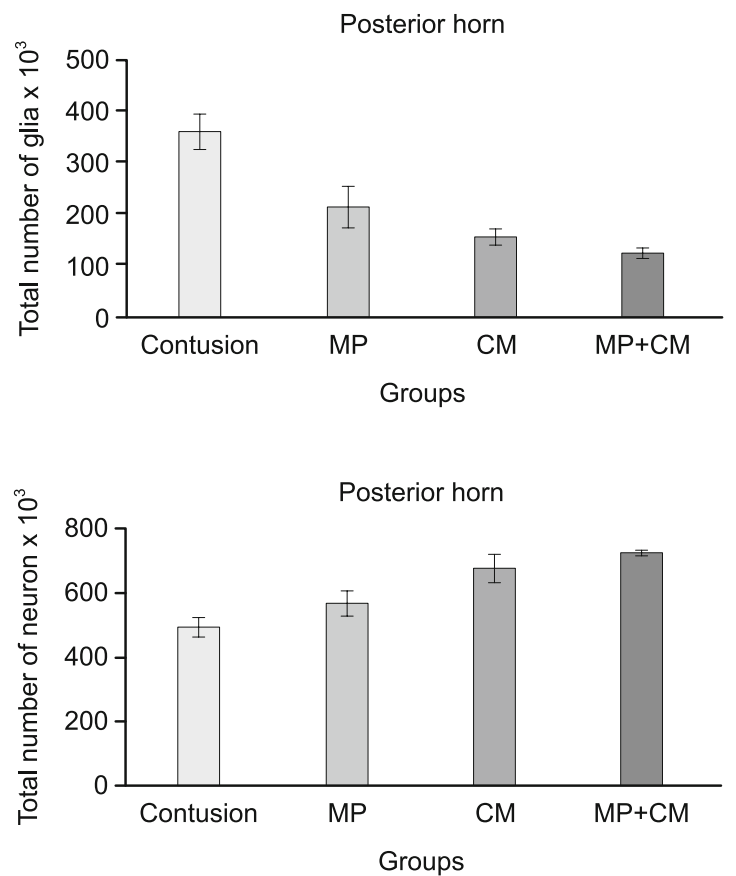

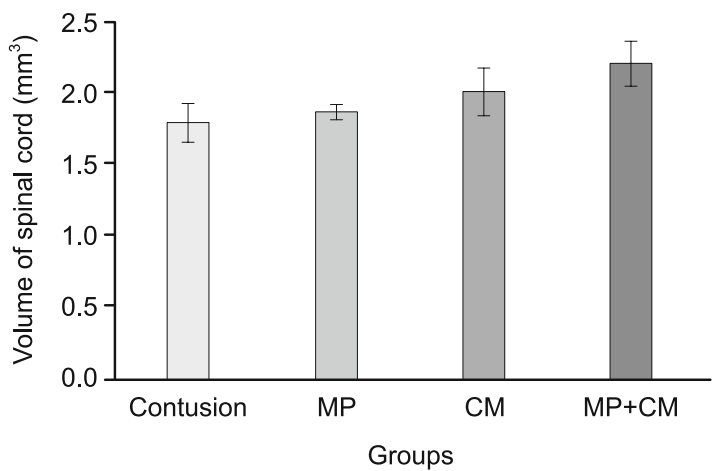

Fig. 7. The results showed that the volume in the MP $+\mathrm{CM}$ group was higher than in the other groups (MP, CM, CO) (*p<0.05). SH: sham group, CO: contusion only as control group, MP: contusion + MP (Pharmacia; $30 \mathrm{mg} / \mathrm{kg}$ i.v. intramuscular injection), CM: contusion + CM and MP + CM: contusion + MP + CM.

nous thrombosis, osteoporosis, pressure ulcers, and neuropathic symptoms. Spinal cord injuries cause breakthrough of the bloodbrain barrier and release of inflammatory factors and activation of glial cells that ultimately cause a spinal cord necrosis (1). After a cascade of lesions, signaling occurs and activates cycles of inflammatory cytokines and hemopoesis that cause apoptosis, demyelination, loss of exons, and degeneration. The inflammation process initially begins with the onset of immune cells and lymphocytes, neutrophils and monocytes to the lesion site. These cells destroy digestive remnants and remove dead cells (2). Our result
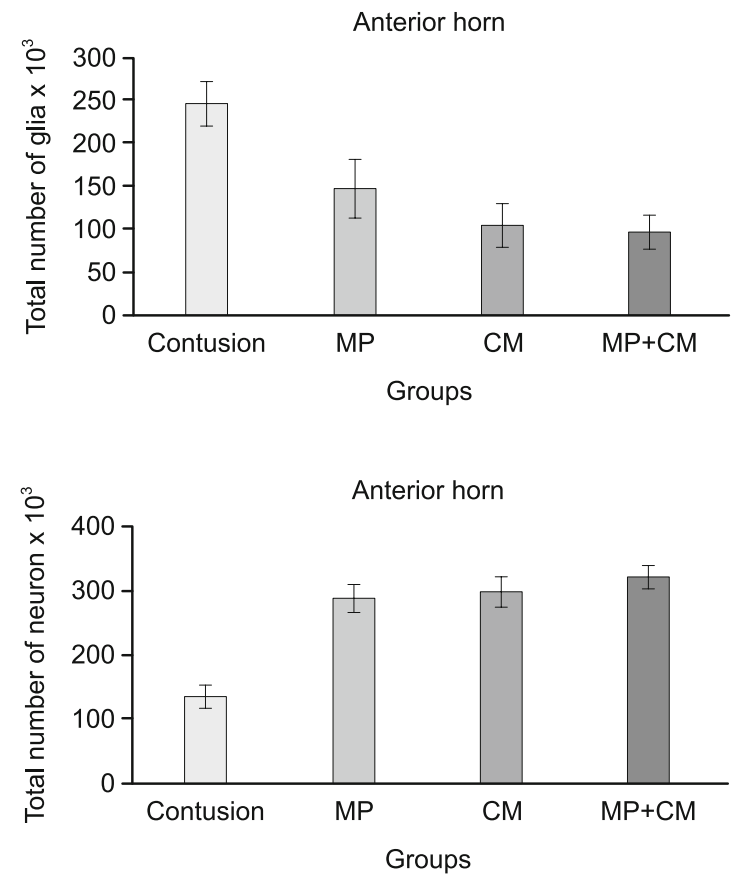

Fig. 8. The results showed that the number of glial cells in the posterior and anterior horns was significantly higher in the control group than in the other experimental groups (MP, CM, MP + CM), and the number of neurons in posterior and anterior horns in the MP + CM group was higher than in the other groups (Contusion, CM, MP) $\left({ }^{*} \mathbf{p}<0.05\right)$. SH: sham group, CO: contusion only as control group, MP: contusion + MP (Pharmacia; $30 \mathrm{mg} / \mathrm{kg}$ i.v. intramuscular injection), CM: contusion $+\mathrm{CM}$ and MP + CM: contusion $+\mathrm{MP}+\mathrm{CM}$. 
showed that a combined effect of MP and CM improved functional recovery and decreases TGF-B expression. We also found that BMSC-CM inhibited neuron and glial cell death and improved functional recovery. Cantinieaux D showed the use of BMSC-CM to prevent neuron apoptosis, activation of macrophages and motor improvement in the rat's SCI model due to neurotrophic secretions of BMSC, which were consistent with our results (14). Other researchers also found that the $\mathrm{CM}$ of some other types of stem cells, such as the umbilical cord and Embryonic stem cells, had similar effects to BMSC on spinal cord injury improvement (15).

Inflammation is a reparative response to tissue injury that comprise an immigration of inflammatory cell such as neutrophil, followed by monocyte, macrophage, $\mathrm{T}$ cell and activation of resident glial cell that this response regulated by specific signaling molecule $(6,10)$. Reactive cells produce cytokines such as: TNF- $\alpha$, Interferon, Interleukins, COX2(cyclooxygenase), prostaglandins,prostacyclin and thromboxane that phagocytose fragments of cell, release proteases and Reactive oxygen species(ROS) These substances destroy the cell membrane and cause necrosis of neuroglial cells $(4,6,10)$. One can prevent the expansion of the lesion by using medications that can reduce inflammatory infiltrate. MP is corticosteroid that inhibits lipid peroxidation and reduces inflammatory cell. High-dose MP is used as one of the effective therapies for the treatment of closed spinal cord injuries. Some studies considered the appropriate time for drug injection 8 hours after the spinal cord injury (16). Some experimental studies have not considered the use of high-dose MP for improvement of acute SCI $(17,18)$. Our results revealed that the application of appropriate MP dose after SCI has positive effects on preventing inflammation and cavity size in spinal cord.

Some researchers reported the simultaneous use of methotrexate combined with methylprednisolone, which had prevented the activity of inflammatory cytokines, apoptosis, and increased antioxidants in the spinal cord injury, although they considered neuroprotective effects MP to be more potent than methotrexate (19). TGF- $\beta$ has a complex character in tissue repair after SCI. previous studies have shown that pathological condition in the brain and spinal cord can alter the level of cytokine/ growth factor such as TGF- $\beta$ that is considered a pleiotropic cytokine $(20,21)$. Some studies suggest that TGF- $\beta$ has a key role in modulating inflammatory responses in SCI. Moreover, it can inhibit astroglioisis progression. Increases in the TGF- $\beta$ levels in the gliosis may bring about a physical and chemical block in the way of axons connection (22). The inflammatory response and gliosis after SCI happens through the TGF- $\beta /$ Smad signaling pathway. It is well documented that activation of immune cells and inflammatory processes are extremely important in regeneration of spinal cord following injury (23). TGF- $\beta$ mRNA increases rapidly after spinal cord injury, which is reaching maximum levels 7 days post-injury at the lesion site and declined thereafter. It has also shown that there is increased levels of TGF- $\beta$ mRNA in the both cranial and caudal regions to the injury location $(24,25)$.Cure by the anti- TGF- $\beta$ antibody decreases the gliosis and probably enhances the microglia/ macrophages triggering following injury. TGF- $\beta$ inhibition facilitates the functional recovery after SCI and it is recommended that suppression of TGF- $\beta$ may be sufficient for functional recovery (26). Our results showed that the amount of TGF- $\beta$ increased during the spinal cord injury, but after the combined treatment we observed a decrease in TGF- $\beta$ concentration. Treatment with MP and BMSC-CM also reduced TGF- $\beta$ concentration, but the lowest amounts were found in the combined treatment group.

We can conclude that simultaneous use of MP and CM will improve SCI, reduce inflammation and improve functional recovery. MP+CM are effective in the treatment of SCI through TGF- $\beta 1$ mRNA.

\section{Acknowledgements}

We are thankful for the funding provided by Skull Base Research Center, Loghman Hakim Hospital, Shahid Beheshti University of Medical Sciences, Tehran, Iran.

\section{Refrences}

1. Ankeny DP, Popovich PG. Mechanisms and implications of adaptive immune responses after traumatic spinal cord injury. Neurosci 2009; 158 (3): 1112-1121.

2. Tica J, Bradbury EJ, Didangelos A. Combined Transcriptomics, Proteomics and Bioinformatics Identify Drug Targets in Spinal Cord Injury. Internat J Mol Sci 2018; 19 (5): 1461.

3. Sayer FT, Kronvall E, Nilsson OG. Methylprednisolone treatment in acute spinal cord injury: the myth challenged through a structured analysis of published literature. Spine J 2006; 6 (3): 335-343.

4. Rosado IR, Lavor MSL, Alves EG, Fukushima FB, Oliveira KM, Silva CMO et al. Effects of methylprednisolone, dantrolene, and their combination on experimental spinal cord injury. Internat J Clin Exp Pathol 2014; 7 (8): 4617.

5. Can M, Gul S, Bektas S, Hanci V, Acikgoz S. Effects of dexmedetomidine or methylprednisolone on inflammatory responses in spinal cord injury. Acta Anaesthesiol Scand 2009; 53 (8): 1068-1072.

6. Bartholdi D, Schwab ME. Expression of pro-inflammatory cytokine and chemokine mRNA upon experimental spinal cord injury in mouse: An in situ hybridization study. Eur J Neurosci 1997; 9 (7): 1422-1438.

7. Kim DK, Kweon KJ, Kim P, Kim HJ, Kim SS, Sohn NW et al. Ginsenoside Rg3 Improves Recovery from Spinal Cord Injury in Rats via Suppression of Neuronal Apoptosis, Pro-Inflammatory Mediators, and Microglial Activation. Molecules 2017; 22 (1): 122.

8. Badner A, Vawda R, Laliberte A, Hong J, Mikhail M, Jose A et al. Early intravenous delivery of human brain stromal cells modulates systemic inflammation and leads to vasoprotection in traumatic spinal cord injury. Stem Cells Translat Med 2016; 5 (8): 991-1003.

9. Beck KD, Nguyen HX, Galvan MD, Salazar DL, Woodruff TM, Anderson AJ. Quantitative analysis of cellular inflammation after traumatic spinal cord injury: evidence for a multiphasic inflammatory response in the acute to chronic environment. Brain 2010; 133 (2): 433-447.

10. Darabi S, Tiraihi T, Delshad A, Sadeghizadeh M, Taheri T, Hassoun HK. Creatine Enhances Transdifferentiation of Bone Marrow Stromal Cell-Derived Neural Stem Cell Into GABAergic Neuron-Like Cells Characterized With Differential Gene Expression. Mol Neurobiol. 2017;54(3):1978-91.

11. Elisa Garcia, Jorge Aguilar-Cevallos, Raul Silva-Garcia, Antonio Ibarra. Cytokine and Growth Factor Activation In Vivo and In Vitro after Spinal Cord Injury. Mediators Inflamm 2016; 2016: 9476020 
12. Cabrera-Aldana EE, Ruelas F, Aranda C, Rincon-Heredia R, Martínez-Cruz A, Reyes-Sánchez A et al. Methylprednisolone Administration Following Spinal Cord Injury Reduces Aquaporin 4 Expression and Exacerbates Edema. Mediat Inflamm 2017; 2017.

13. Evaniew N, Noonan VK, Fallah N, Kwon BK, Rivers CS, Ahn H et al. Methylprednisolone for the treatment of patients with acute spinal cord injuries: a propensity score-matched cohort study from a Canadian multi-center spinal cord injury registry. J Neurotrauma 2015; 32 (21): 1674-1683.

14. Peyvandi A.A, Abbaszadeh H.-A, Roozbahany N.A, Pourbakht A, Khoshsirat S, Niri HH, Peyvandi H, Niknazar S. Deferoxamine promotes mesenchymal stem cell homing in noise-induced injured cochlea through PI3K/AKT pathway. Cell Prolif. 2018 Apr;51(2):e12434.

15. Abbaszadeh H A, Tiraihi T, Sadeghi Y, Delshad A R, Sadeghizadeh M, Taheri T et al. Decrease in Cavity Size and Oligodendrocyte Cell Death Using Neurosphere-Derived Oligodendrocyte-Like Cells in Spinal Cord Contusion Model. IBJ 2018; 22 (4): 246-257.

16. Shams Nooraei M, Noori-Zadeh A, Darabi S, Rajaei F, Golmohammadi Z, Abbaszadeh HA. Low Level of Autophagy-Related Gene 10 (ATG10) Expression in the 6-Hydroxydopamine Rat Model of Parkinson's Disease. Iran Biomed J. 2018;22(1):15-21.

17. Guo L, Rolfe AJ, Wang X, Tai WJ, Cheng ZJ et al. Rescuing macrophage normal function in spinal cord injury with embryonic stem cell conditioned media. Mol Brain 2016; 9: 48.

18. Hugenholtz H, Cass DE, Dvorak MF, Fewer DH, Fox RJ, Izukawa DM, Lexchin $\mathbf{J}$ et al. High-dose methylprednisolone for acute closed spinal cord injury-only a treatment option. Can J Neurol Sci 2002; 29 (3): $227-235$.
19. Cheung V, Hoshide R, Bansal V, Kasper E, Chen CC. Methylprednisolone in the management of spinal cord injuries: Lessons from randomized, controlled trials. Surg Neurol Int 2015; 6: 142. doi: 10.4103/21527806.163452. eCollection 2015.

20. Cabrera-Aldana EE, Ruelas F, Aranda C, Rincon-Heredia R, Martínez-Cruz A et al. Methylprednisolone Administration Following Spinal Cord Injury Reduces Aquaporin 4 Expression and Exacerbates Edema. Mediators Inflamm 2017; 2017: 4792932. doi: 10.1155/2017/4792932.

21. Liu JT, Zhang S, Gu B, Li HN, Wang SY, Zhang SY. Methotrexate combined with methylprednisolone for the recovery of motor function and differential gene expression in rats with spinal cord injury. Neural Regen Res 2017; 12 (9): 1507-1518. doi: 10.4103/1673-5374.215263.

22. Dobolyi A, Vincze C, Pál G, Lovas G. The Neuroprotective Functions of Transforming Growth Factor Beta Proteins. Int J Mol Sci 2012; 13 (7): 8219-8258.

23. Hsu JYC, Bourguignon LYW, Adams CM, Peyrollier K, Zhang HQ et al. Matrix metalloproteinase-9 facilitates glial scar formation in the injured spinal cord. J Neurosci 2008; 28 (50): 13467-13477.

24. Kim SY, Senatorov Jr. VV, Morrissey CS, Lippmann K et al. TGF $\beta$ signaling is associated with changes in inflammatory gene expression and perineuronal net degradation around inhibitory neurons following various neurological insults. Sci Report 2017; 7, Article number: 7711.

25. Church JS, Kigerl KA, Lerch JK, Popovich PG, McTigue DM. TLR4 Deficiency Impairs Oligodendrocyte Formation in the Injured Spinal Cord. J Neurosci 2016; 36 (23): 6352-6364.

26. Li SY, Gu XS, Yi S.The Regulatory Effects of Transforming Growth Factor- $\beta$ on Nerve Regeneration. Cell Transplantat 26 (3): 381-394.

Received July 16, 2018. Accepted August 20, 2018. 